\title{
Modificación de Betún-Venezuela por reacción de Friedel y Crafts
}

\author{
MANUEL BLANCO, Dr. en Ciencias Químicas \\ ANGEL CUEVAS, Lcdo. en Ciencias Químicas \\ JUAN J. ORTEGA, Dr. en Química Industrial \\ Area de Materiales Orgánicos del Laboratorio Central de Estructuras y Materiales.

\section{RESUMEN}

En el presente trabajo se modificó un betún-Venezuela, de naturaleza newtoniana, mediante reacción de Friedel y Crafts, transformándolo en unos nuevos materiales, ya de carácter pseudoplástico, con unas propiedades notablemente distintas a las del producto de partida. Los nuevos materiales son más duros y de menor susceptibilidad térmica, lo que los hace útiles para pavimentación e impermeabilización. Las modificaciones se han realizado con cauchos, policloruro de vinilo, anhidrido ftálico, anhidrido maléico, 1,2-propanodiol, hexametilendiisocianato y toluen-2,4-diisocianato.

\section{SUMMARY}

In this work, a Venezuelan-bitumen of newtonian nature was modified by Friedel and Crafts reaction, changing it in new materials that present pseudoplastic characteristics, with very different properties from the original product. These new materials are harder and have less thermical susceptibility and allow us to use them in many applications such as paving and roofing. The modifications have been made with rubbers, PVC, phtalic anhydride, maleic anhydride, 1, 2-propyleneglycol, hexamethylenediisocyanate and toluylene-2,4-diisocyanate.

\section{INTRODUCCION}

El betún asfáltico es el residuo que queda en la destilación del petróleo y, quizás, el primer material termoplástico utilizado por el hombre. Aparte de su utilización en pavimentación, su principal aplicación es la impermeabilización, desde cubiertas de edificios hasta la de embalses, presas, túneles, tableros de puentes, etc. Como tal material termoplástico, el betún tiene una importante desventaja: es muy sensible a los cambios de temperatura. Tiende a hacerse duro y frágil en tiempo frío, mientras que en épocas calurosas se reblandece y fluye. Las diversas técnicas que normalmente se han utilizado para salvar estas deficiencias han creado, frecuentemente, nuevos problemas. Por ejemplo, al mezclar el betún con plastificantes para aumentar su resistencia a las bajas temperaturas, se obtiene un producto de menor resistencia a las temperaturas elevadas. Inversamente el uso de un "filler" para mejorar la resistencia a altas temperaturas se traduce en un aumento de la fragilidad a temperaturas bajas.

Por lo anteriormente expuesto, la modificación de los betunes asfálticos parece una necesidad general: se debe mejorar la susceptibilidad térmica así como sus propiedades reológicas y mecánicas. Este proceso puede llevarse a cabo mediante una adición de determina- dos productos: azufre, copolímeros y elastómeros, o bien por reacción química (1). En este trabajo vamos a circunscribirnos a las modificaciones producidas por reacción de Friedel y Crafts.

El betún asfáltico está formado por un conjunto de hidrocarburos aromáticos polisustituidos a los que acompañan fundamentalmente alcanos, cicloalcanos y alquenos. Muchos investigadores como Yen (2) demostraron por RMN que había hidrógenos aromáticos sin sustituir capaces de reaccionar con distintas sustancias en presencia de catalizadores ácidos mediante una reacción de sustitución electrófila $(3,4)$ :

Según Epinat (5) la reacción de Friedel y Crafts aplicada a los betunes asfálticos puede ocurrir según los esquemas que se presentan a continuación con dos tipos de productos.

a) Compuestos macromoleculares (poliésteres, poliestirenos clorados,...).
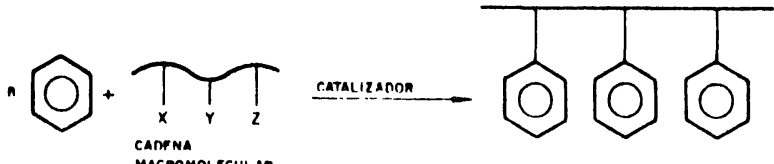
b) Moléculas bifuncionales (dienos, diácidos, dioles, anhídridos cíclicos,...).

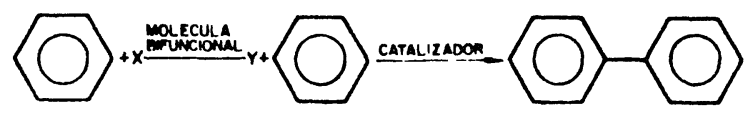

\section{PARTE EXPERIMENTAL}

\section{Reactivos empleados}

Se ha utilizado un betún-Venezuela cuyas carácterísticas se han medido convenientemente con objeto de poder posteriormente comprobar las transformaciones habidas en el mismo.

Como catalizador de la reacción de Friedel y Crafts hemos empleado el clásico tricloruro de aluminio anhidro, de elevado grado de pureza.

Entre los agentes alquilantes y acilantes se ha hecho uso de dos tipos de productos:

\section{Compuestos macromoleculares:}

- Poli (cloruro de vinilo) en polvo y sin plastificar.

- Caucho-B de butadieno-estireno, con un $30 \%$ de poliestireno, peso molecular típico $3 \cdot 10^{5}$, peso específico 0,94 y dureza Shore-A, 80 .

- Caucho-C, un elastómero de base poliolefínica, suministrado por la firma Tecnopolimeri de Milán.

\section{Moléculas bifuncionales:}

- Andídrido ftálico.

- Andídrido maléico.

- 1,2-propanodiol.

- Hexametilendiisocianato.

- Toluen-2,4-diisocianato.

\section{Procedimiento operatorio}

La reacción se ha llevado a cabo en un reactor donde se colocaron el betún y el tricloruro de aluminio anhidro, a una temperatura de $160^{\circ} \mathrm{C}$, en atmósfera inerte de nitrógeno. Poco a poco se ha añadido el agente alquilante o acilante, manteniendo la reacción con agita-
TABLA 1

Proporciones de reactivos introducidas al reactor para la modificación de Betún-Venezuela con compuestos macromoleculares

\begin{tabular}{|c|c|c|c|c|c|c|c|}
\hline \multirow{2}{*}{ 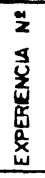 } & \multicolumn{6}{|c|}{ X EN PESO } & \multirow{2}{*}{ 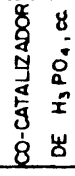 } \\
\hline & 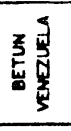 & $\stackrel{\text { Za }}{a}$ & 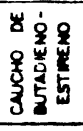 & 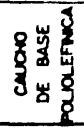 & 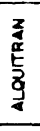 & 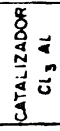 & \\
\hline 1 & 93 & 3 & - & - & - & 2 & 1 \\
\hline 2 & 96 & - & 2 & - & - & 2 & 1 \\
\hline 3 & 94 & - & - & 3 & 1 & 2 & 0,3 \\
\hline 4 & 93 & - & - & 3 & 2 & 2 & 0,3 \\
\hline
\end{tabular}

TABLA II

Proporciones de reactivos introducidas al reactor para la modificación de Betún-Venezuela con moléculas bifuncionales

\begin{tabular}{|c|c|c|}
\hline 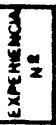 & $\begin{array}{c}\text { ACENTE } \\
\text { MQURANTE } 0 \\
\text { ACILANTE }\end{array}$ & $\begin{array}{l}\text { RELACION EN \% EN } \\
\text { PESO DE AGENTE } \\
\text { NLOUILANTE O } \\
\text { ACILANTE/ } a_{3} \text { ML }\end{array}$ \\
\hline 5 & \multirow{8}{*}{$\begin{array}{l}3 \\
\frac{3}{4} \\
\frac{3}{2} \\
8 \\
\frac{8}{3} \\
\frac{3}{2} \\
\frac{2}{4}\end{array}$} & 10 \\
\hline 6 & & $5: 0$ \\
\hline 7 & & $0: 1$ \\
\hline 9 & & $0,5: 1$ \\
\hline 9 & & $1: 1$ \\
\hline 10 & & $5: 1$ \\
\hline 11 & & 0.2 \\
\hline 12 & & 0.52 \\
\hline$i 3$ & \multirow{8}{*}{$\begin{array}{l}8 \\
\frac{8}{3} \\
\frac{1}{5} \\
4 \\
8 \\
\frac{8}{6} \\
\frac{3}{6} \\
\frac{3}{3}\end{array}$} & $1: 0$ \\
\hline 4 & & $5: 0$ \\
\hline 15 & & $0: 1$ \\
\hline 16 & & $0,5: 1$ \\
\hline 17 & & $1: 1$ \\
\hline 10 & & 3:1 \\
\hline 1 & & $0: 2$ \\
\hline 20 & & $0.5: 2$ \\
\hline 21. & \multirow{8}{*}{$\begin{array}{l}8 \\
8 \\
5 \\
5 \\
0 \\
\vdots \\
\vdots \\
\vdots \\
=\end{array}$} & 10 \\
\hline 22 & & 5:0 \\
\hline 23 & & $0: 1$ \\
\hline 24 & & $0.5: 1$ \\
\hline 25 & & $1: 1$ \\
\hline 20 & & $5: 1$ \\
\hline 27 & & 0.2 \\
\hline 28 & & $0,5: 2$ \\
\hline 29 & \multirow{8}{*}{ 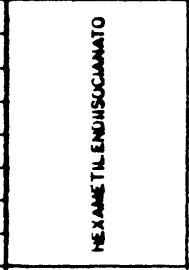 } & $1: 0$ \\
\hline 30 & & 5:0 \\
\hline 31 & & $0: 1$ \\
\hline 32 & & $0,5: 1$ \\
\hline 33 & & $1: 1$ \\
\hline 34 & & 3:1 \\
\hline 35 & & 0.2 \\
\hline 36 & & $0,5 \cdot 2$ \\
\hline 37 & \multirow{6}{*}{ 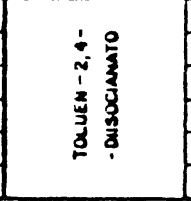 } & $1: 1$ \\
\hline 38 & & 3.1 \\
\hline 39 & & 3:1 \\
\hline 40 & & 1.2 \\
\hline 41 & & 3.2 \\
\hline 42 & & $3: 2$ \\
\hline
\end{tabular}


ción constante durante 16 horas. La temperatura no sobrepasó en ningún momento los $160^{\circ} \mathrm{C}$ para evitar el proceso de sublimación del catalizador que tiene lugar a $180^{\circ} \mathrm{C}$.

Las cantidades de reactivos utilizadas para llevar a cabo la incorporación de los compuestos macromoleculares al betún asfáltico objeto de estudio figuran en la tabla I, mientras que en la tabla II aparecen las proporciones iniciales introducidas al reactor cuando la reacción tiene lugar con moléculas bifuncionales.

La dificultad de incorporación de los cauchos poliolefínicos ha sido obviada con la presencia de un pequeño porcentaje de alquitrán.

Para constatar la eficacia del catalizador en el proceso reactivo se han hecho experiencias sin el concurso del mismo (Experiencias números $5,6,13,14,21,22,29$ y 30). Al tiempo que en otros casos (Experiencias números 7 , $11,15,19,23,27,31$ y 35) no se utilizó agente alquilante 0 acilante, es decir, que la reacción se limitaba al contacto betún-catalizador, pues sabido es que los catalizadores ácidos de Friedel y Crafts actúan sobre los compuestos aromáticos, produciendo isomerizaciones, desalquilaciones, transposiciones, etc. $(6,7)$.

Los ensayos realizados para la obtención de las propiedades que caracterizan a este tipo de productos, tanto original como finales, se han hecho siguiendo las directrices de la bibliografía internacional sobre el tema (8). En cuanto a las propiedades reológicas, hemos hecho uso de un reómetro capilar, tipo Instron3211 de gran precisión. Se determinaron las características reológicos entre 50 y $80^{\circ}$, con velocidades de deformación comprendidas entre 1,77 y $2.362,2 \mathrm{~s}^{-1}$.

El envejecimiento de los productos que estamos considerando se realizó por el procedimiento de "película delgada" (9) sometiéndolos a calefacción en una estufa a $163 \pm 1^{\circ} \mathrm{C}$ durante 5 horas. Para ello se hizo uso de unas bandejas apropiadas donde los materiales se colocaban con un espesor de $3 \mathrm{~mm}$.

\section{RESULTADOS}

Las características tecnológicas del betún original así como de los productos modificados con sustancias de tipo macromolecular se presentan en la tabla III.

Las tablas IV, V, VI y VII, muestran, respectivamente, las características de los nuevos materiales obtenidos en la modificación de betúnVenezuela, en presencia de tricloruro de aluminio, con anhídridos, 1,2-propanodiol, hexametilendiisocianato y toluen-2, 4-diisocianato.

TABLA III

Características tecnológicas de los productos obtenidos en la modificación química de Betún-Venezuela con sustancias macromoleculares

\begin{tabular}{|c|c|c|c|c|c|c|c|}
\hline \multirow{3}{*}{ CARACTERISTICA } & & & \multicolumn{5}{|c|}{ PRODUCTOS INCORPORADOS AL BETUN } \\
\hline & \multicolumn{2}{|c|}{$\begin{array}{c}\text { BETUN } \\
\text { VENEZUELA }\end{array}$} & $\begin{array}{l}P Y C(5 \%) \\
\mathrm{AC}_{3}(2 \%) \\
\mathrm{H}_{3} \mathrm{PO}_{4} 1 \mathrm{ISC}\end{array}$ & \multicolumn{2}{|c|}{$\begin{array}{l}\text { CAUCHO-B }\left(2 \%_{6}\right) \\
\text { AL Cl }\left(2 x_{1}\right) \\
\mathrm{H}_{3} \mathrm{PO}_{4} \mid \mathrm{CC}\end{array}$} & $\begin{array}{l}\text { CAUCHO-C }\left(3 x_{)}\right. \\
\text {MOUITRAN }\left(1 x_{)}\right. \\
A\left(C_{3}\left(2 x_{)}\right.\right. \\
H_{3} P O_{4}(0,3 \%)\end{array}$ & 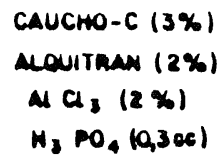 \\
\hline & ORIG. & ENV. & ORIGINAL & ORIG. & ENV. & ONIBIAAL & ORIGINAL \\
\hline $\begin{array}{l}\text { PUNTO DE REGLANDECIMIENTO } \\
\text { (ANILLO Y BOLA), •C }\end{array}$ & 52 & 65 & 88 & 67 & 62 & 80 & 63 \\
\hline $\begin{array}{l}\text { PENETRACION }\left(25^{\circ} \mathrm{C}, 1000.5 \mathrm{~s}\right) \\
\text { Docimos milimeiro }\end{array}$ & 63 & 35 & 26 & 39 & 28 & 21 & 26 \\
\hline INDICE DE PENETHACION & $-0,2$ & 1,2 & 3,9 & 1,6 & 0,2 & 2,2 & 3,2 \\
\hline ROTURA FRAAS, 'C & -9 & $-7,7$ & -7 & $-4,5$ & $-1,5$ & 0 & - \\
\hline DUCTILIOAD A $25^{\circ} \mathrm{C}, \mathrm{cm}$. & $>114$ & $>114$ & 4 & 93 & so & - & - \\
\hline PESO ESPECIFICO & 1,03 & 1,04 & 1,03 & 1,05 & 1,04 & - & - \\
\hline
\end{tabular}


TABLA IV

Propiedades tecnológicas de los productos modificados obtenidos por reacción de Friedel y Crafts entre Betún-Venezuela y los anhídridos maleico y ftalico

\begin{tabular}{|c|c|c|c|c|c|c|c|c|c|c|c|c|c|c|c|c|c|c|c|}
\hline \multirow{3}{*}{ CARACTERISTICA } & \multirow{3}{*}{$\begin{array}{ll}z & \frac{1}{4} \\
3 & \frac{z}{2} \\
\frac{w}{4} & \frac{0}{g} \\
0 & \frac{1}{8}\end{array}$} & \multicolumn{3}{|c|}{ RELACION } & N $\%$ & , DE & \multicolumn{3}{|c|}{ ANHIDFIDO } & \multicolumn{4}{|c|}{ DE } & \multicolumn{2}{|c|}{ ALMIN"O, } & WDH & D N & \multicolumn{2}{|c|}{ EETUN } \\
\hline & & \multicolumn{5}{|c|}{ ANHIDRLO } & \multicolumn{3}{|c|}{ MALEICO } & & & \multicolumn{4}{|c|}{ ANHDRDO } & \multicolumn{2}{|c|}{ FTALICO } & & \\
\hline & & $1: 0$ & $5: 0$ & $0: 1$ & $0,5.1$ & $1: 1$ & 5: 1 & $0: 2$ & $0,5.2$ & 1: 2 & $5: 2$ & 1.0 & 50 & $0,5: 1$ & $1: 1$ & 0.1 & a.s.2 & $1: 2$ & 8:2 \\
\hline $\begin{array}{l}\text { PUNTO DE RECLAMOECIMIENTO } \\
\text { (ANILLO Y DOLA), •C }\end{array}$ & 52 & 57 & 60 & 65 & 68 & 71 & 71 & 64 & 75 & 78 & 96 & 57 & 80 & 70 & 68 & 64 & 70 & 80 & 10 \\
\hline $\begin{array}{l}\text { PENETRACION }\left(25^{\circ} \mathrm{C}, 100 \mathrm{~s} .8 .\right) \\
\text { Decimes millmetre }\end{array}$ & 63 & 46 & 39 & 33 & 27 & 25 & 21 & 29 & 20 & 17 & 15 & 55 & 50 & 27 & 25 & 30 & 20 & 10 & 20 \\
\hline INDICE DE PENETRACION & $-0,2$ & 0 & 0,3 & 1,0 & 1,1 & 1,3 & 1,0 & 0,4 & 1,4 & 1,6 & 3,4 & $-0,6$ & 0,2 & 1,4 & 1,0 & 0,6 & 1,7 & 20 & 2,2 \\
\hline ROTURA FRAAS, •C & -9 & -5 & -6 & -6 & -5 & -2 & 3 & -7 & 3 & 0 & -4 & -5 & -7 & -5 & 0 & -3 & 0 & -2 & -1 \\
\hline
\end{tabular}

TABLA V

Características tecnológicas del Betún-Venezuela modificado con 1,2-propanodiol por reacción de Friedel y Crafts

\begin{tabular}{|c|c|c|c|c|c|c|c|c|c|}
\hline \multirow{2}{*}{ CARACTERISTICA } & \multirow{2}{*}{$\begin{array}{l}\text { BETUN } \\
\text { ORIGINAL }\end{array}$} & \multicolumn{8}{|c|}{$\begin{array}{l}\text { RELACION, EN \%, DE 1,2 - PROPANODIOL: TRICLORURO } \\
\text { DE ALUMINIO AÑADIDO AL BETUN }\end{array}$} \\
\hline & & $1: 0$ & $5: 0$ & $0,5: 1$ & $1: 1$ & $5: 1$ & $0,5: 2$ & $1: 2$ & $5: 2$ \\
\hline $\begin{array}{l}\text { PUNTO DE REBLANDECIMIENTO } \\
\text { (ANILLLO Y OOLA), •C }\end{array}$ & 52 & 55 & 53 & 64 & 67 & 60 & 73 & 72 & 62 \\
\hline $\begin{array}{l}\text { PENETRACION }\left(25^{\circ} \mathrm{C}, 100 \mathrm{~s} .8 \mathrm{~s}\right) \\
\text { Decimes millimelre }\end{array}$ & 63 & 55 & 63 & 30 & 28 & 50 & 26 & 25 & 37 \\
\hline IMOICE DE RENETRACION & $-0,2$ & 0,2 & $-0,1$ & 0,6 & $-0,9$ & 1,2 & 1,7 & 1,6 & 1,3 \\
\hline ROTURA FRAAS, ${ }^{\circ} \mathrm{C}$ & -9 & -5 & -6 & -2 & 0 & -3 & -4 & -4 & -2 \\
\hline
\end{tabular}

TABLA VI

Propiedades tecnológicas de los productos obtenidos en la modificación de Betún-Venezuela con hexametilendiisocianato, mediante reacción de Friedel y Crafts

\begin{tabular}{|c|c|c|c|c|c|c|c|c|c|}
\hline \multirow{2}{*}{ CARACTERISTICA } & \multirow{2}{*}{$\begin{array}{l}\text { BETUN } \\
\text { ORIGINAL }\end{array}$} & \multicolumn{8}{|c|}{$\begin{array}{c}\text { RELIACION, EN \%, DE HEXAMETILENDIISOCIANATO : TRICLORURO } \\
\text { DE ALUMINIO AÑADIDO AL BETUN }\end{array}$} \\
\hline & & $1: 0$ & $5: 0$ & $0,5: 1$ & $1: 1$ & $5: 1$ & $0,5: 2$ & $1: 2$ & $5: 2$ \\
\hline $\begin{array}{l}\text { PUNTO DE REBLANDECIMIENTO } \\
\text { (ANILLOO Y BOLA), •C }\end{array}$ & 52 & 52 & 43 & 67 & 67 & 65 & 70 & 73 & 79 \\
\hline $\begin{array}{l}\text { PENETRACION }\left(25^{\circ} \mathrm{C}, 100 \mathrm{~g} .58 .\right) \\
\text { Docimas millmolro }\end{array}$ & 63 & 58 & 150 & 29 & 31 & 35 & 29 & 28 & 32 \\
\hline IMOICE DE PENETRACION & $-0,2$ & $-0,4$ & 0,1 & 1,0 & 1,2 & 1,1 & 0,6 & 1,9 & 3,0 \\
\hline ROTUnA FRAAS, ${ }^{\circ} \mathrm{C}$ & -9 & -3 & -5 & -2 & -4 & 1,5 & -4 & -4 & -1 \\
\hline
\end{tabular}


TABLA VII

Resultados obtenidos en los ensayos de carácter tecnológico en los productos de modificación de Betún-Venezuela con toluen-2,4-diisocianato, mediante reacción de Friedel y Crafts

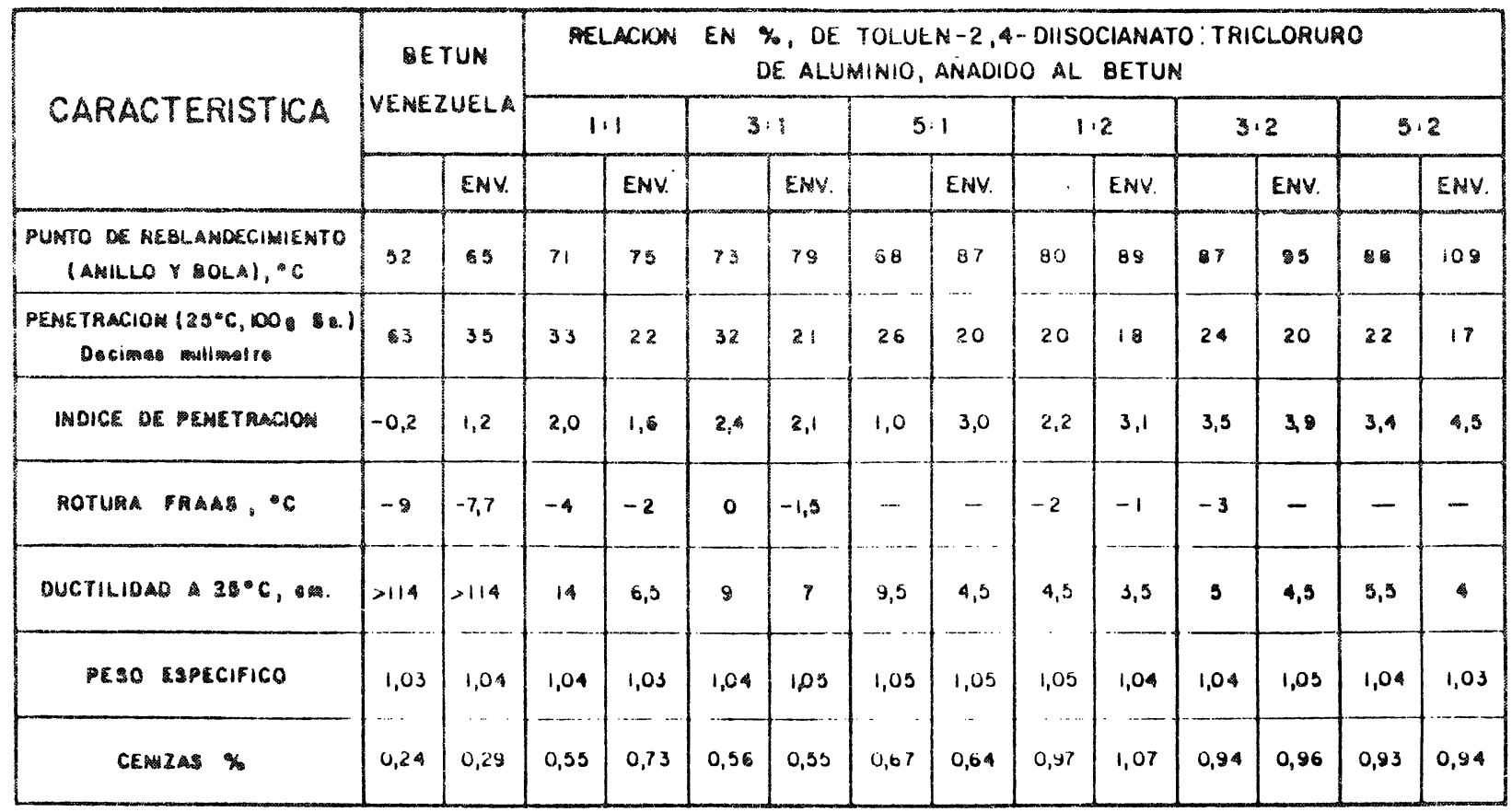

TABLA VIII

Características tecnológicas del Betún-Venezuela modificado con diversas sustancias mediante reacción de Friedel y Crafts

\begin{tabular}{|c|c|c|c|c|c|c|c|c|c|}
\hline \multirow{2}{*}{ CARACTERISTICA } & \multirow{2}{*}{$\begin{array}{l}\text { BETUN } \\
\text { ORIGINAL }\end{array}$} & \multirow{2}{*}{ 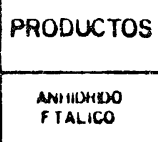 } & \multicolumn{2}{|c|}{ INCORPORADOS AL BETUN } & MEDIANTE & REACCION & \multicolumn{2}{|c|}{ DE FRIEDEL Y } & CRAFTS \\
\hline & & & $\begin{array}{l}\text { ANHWWHUO } \\
\text { MALEKO }\end{array}$ & 1,2 andoratar & 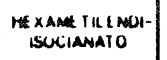 & $\begin{array}{l}\text { TOL UEN-2 4- } \\
\text { OWSOCLAANITO }\end{array}$ & pre & caucro- & cancroo-c \\
\hline $\begin{array}{l}\text { PUNTO DE REBLAMOECIMIENTO } \\
\text { (ANILLO Y BOLA), 'C }\end{array}$ & 52 & 58 & 71 & 67 & 67 & 71 & 80 & 67 & 0 \\
\hline $\begin{array}{l}\text { PEMETRACHON }\left(25^{\circ} \mathrm{C}, 100.58 .\right) \\
\text { Dosimes millienatire }\end{array}$ & 63 & 23 & 25 & 28 & 31 & 33 & 26 & 39 & 21 \\
\hline INDICE OE PEMETRACION & $-0,2$ & 1,0 & 1,3 & $-0,9$ & 1,2 & 2,0 & 3,8 & 1,8 & 2,2 \\
\hline ROTURA FAAAS, "C & -9 & 0 & -2 & 0 & -4 & -4 & -7 & $-4,5$ & 0 \\
\hline DUCTILIDAD \& $25^{\circ} \mathrm{C}, \mathrm{cm}$. & $>114$ & - & - & - & - & 14 & 4 & 23 & - \\
\hline PESO ESPECIFICO & 1,03 & - & - & - & - & 1,04 & 1,03 & 1,05 & - \\
\hline
\end{tabular}

Asimismo, en la tabla VIII y con objeto comparativo se da cuenta de los resultados tecnológicos habidos al hacer reaccionar al betún original con distintos agentes alquilantes y acilantes. En todos los casos la relación, en \% en peso de agente modificador/catalizador, ha sido de 1:1, excepto en el caso del PVC y Caucho-B, pues sólo se disponía de una com- posición. Para la comparación con Caucho- $\mathrm{C}$ se ha empleado la denominada experiencia n. ${ }^{\circ} 3$.

En la figura 1 se puede observar el diagrama reológico del betún-Venezuela, de naturaleza newtoniana, donde la velocidad de deformación varía linealmente con la tensión. Las figu- 
ras 2 y 3 muestran, respectivamente, los diagramas del betún-Venezuela modificado por reacción de Friedel y Crafts con toluen-2,4-diisocianato, en proporción en peso, diisocianato/tricloruro de aluminio $1: 1$, original y envejecido.

La viscosidad ha aumentado de un modo muy considerable con relación al betún original, como se puede apreciar en las figuras 4 y 5 , donde representamos la viscosidad, en poises, frente a la temperatura de ensayo, en grados centígrados.

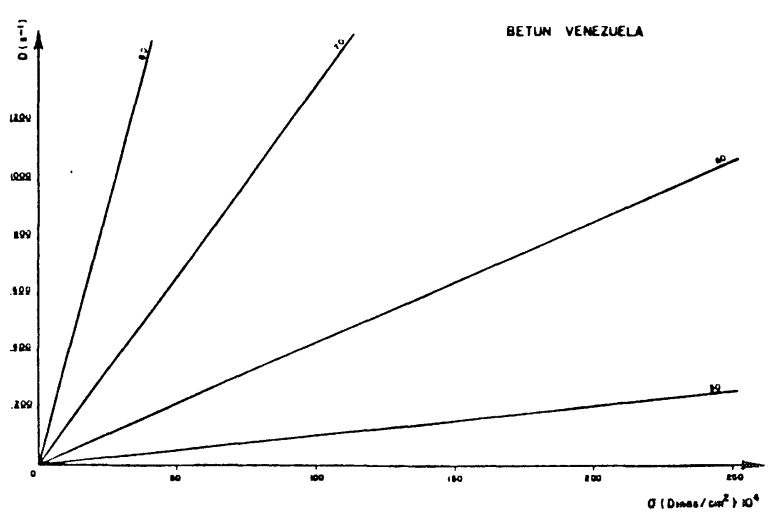

Fig. 1

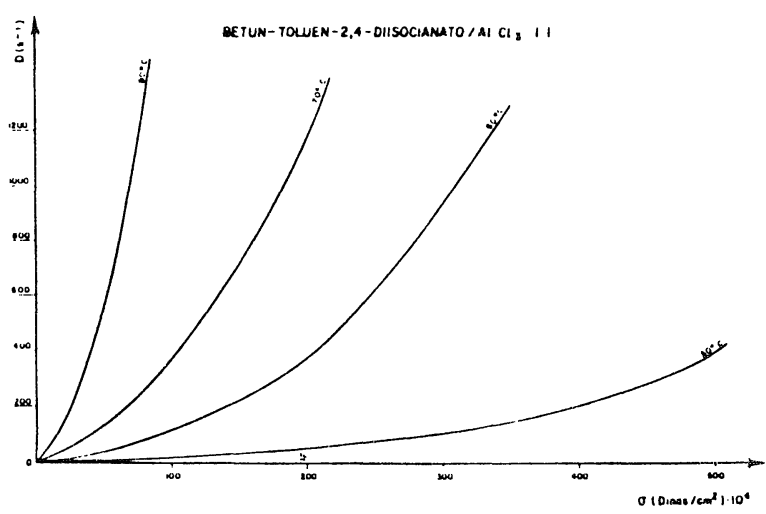

Fig. 2

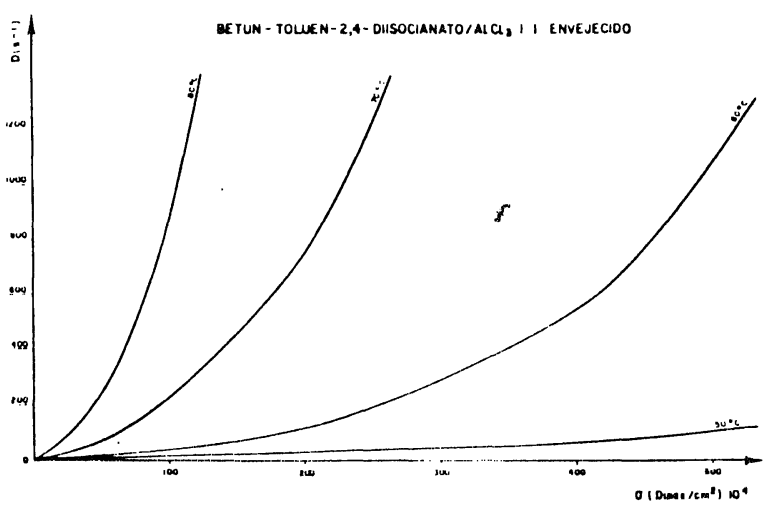

Fig. 3

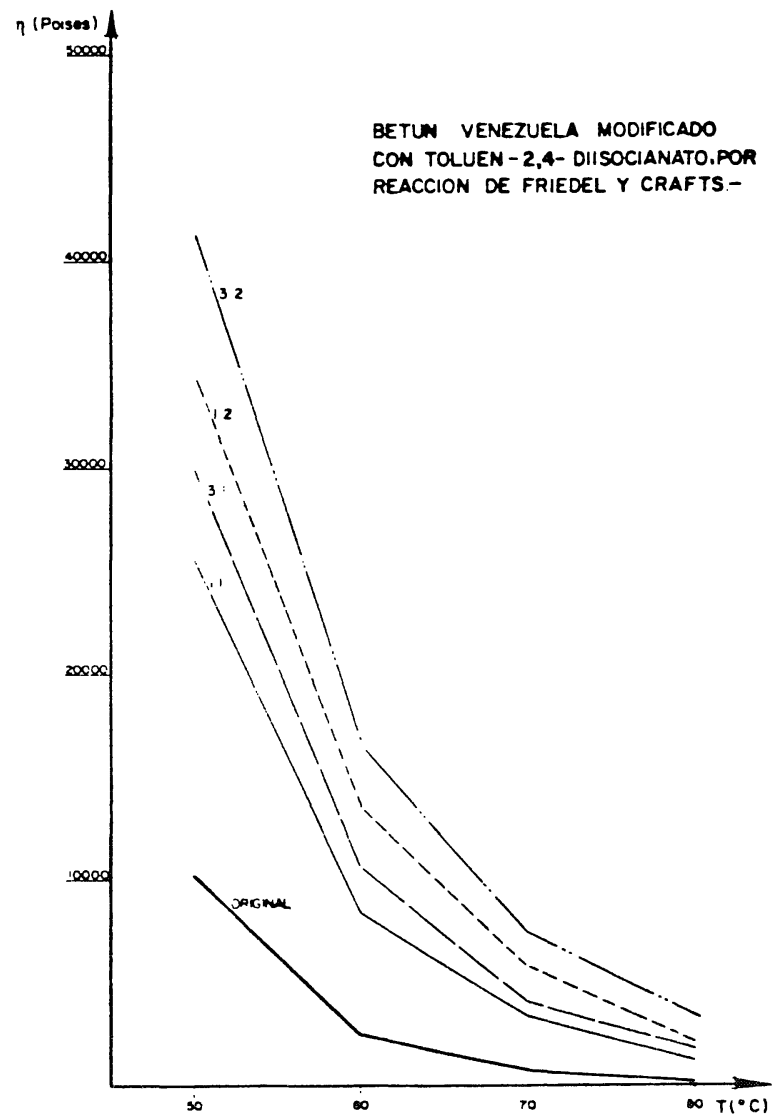

Fig. 4

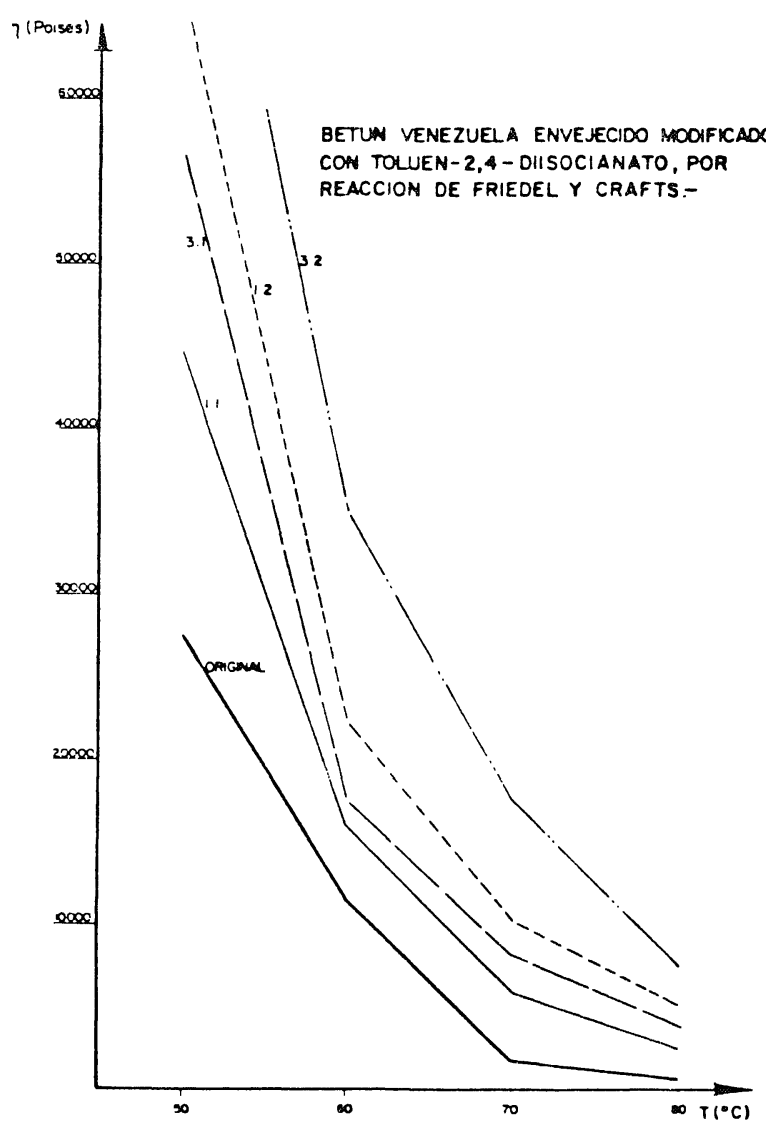

Fig. 5

MATERIALES DE COSTRUCCION, Vol. 37, n.²05, enero/febrero/marzo 1987 
Si la representación viscosidad-temperatura, se lleva a cabo en papel doble logarítmico se obtienen unas rectas cuya pendiente es la denominada susceptibilidad térmica, es decir, la resistencia que presenta el material a cambiar su viscosidad con la variación de la temperatura. La disminución de la susceptibilidad térmica era uno de los objetivos fundamentales de nuestro trabajo. De los datos experimentales se han obtenido unas gráficas cuyas pendientes tabulamos en IX, donde se puede comprobar que dicho parámetro ha sido reducido de una forma muy notable con respecto al betún original.

TABLA IX

Susceptibilidad térmica del Betún-Venezuela $y$ de los productos obtenidos por reacción de Friedel y Crafts con toluen-2,4-diisocianato

\begin{tabular}{|c|c|c|c|}
\hline \multirow{2}{*}{\multicolumn{2}{|c|}{ MATERIAL }} & \multicolumn{2}{|c|}{$\begin{array}{c}\text { SUSCEPTIBLIDAO TERMICA } \\
\text { DE PRODUCTOS }\end{array}$} \\
\hline & & ORIGINALES & ENVEJECIOOS \\
\hline \multicolumn{2}{|c|}{$\begin{array}{c}\text { 3ETUN- } \\
\text { VENEZUELA } \\
\text { SIN } \\
\text { WOOIFICAR }\end{array}$} & $-7,527$ & $-7,552$ \\
\hline \multirow{4}{*}{ 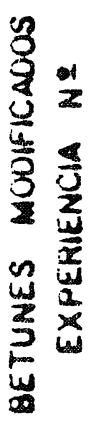 } & 39 & $-6,104$ & $-6,035$ \\
\hline & 40 & $-3,699$ & $-3,707$ \\
\hline & 41 & $-3,534$ & $-5,643$ \\
\hline & 42 & $-5,310$ & $-5,504$ \\
\hline
\end{tabular}

\section{CONCLUSIONES}

Como conclusiones finales nos parece adecuado citar las siguientes:

1. Los nuevos productos obtenidos, mediante reacción de sustitución electrófila de Friedel y Crafts, son mucho más rígidos que los betunes de partida, como consecuencia de la formación de estructuras complejas entre los aniilos aromáticos de los betunes y los agentes macromoleculares o bifuncionales con los que se han hecho reaccionar.
Dicho aumento de la complejidad del material ha dado lugar, asimismo, a un incremento del índice de penetración, que es otra forma de evaluar la variación de la susceptibilidad térmica; a mayor índice de penetración, menor susceptibilidad térmica.

2. Las reacciones entre el betún y el catalizador, sin la presencia de agentes de alquilación o acilación, condujeron a resultados semejantes, pero sin variaciones tan notables a las citadas en el punto anterior. El motivo radica en las reacciones que tiene lugar en el betún: isomerización, desalquilación, transposición, etc.; o bien por las reacciones entre los componentes aromáticos de dicho betún con los agentes de carácter alifático del mismo, que actuarían como agentes alquilantes en presencia del tricluroro de aluminio.

3. Asimismo, se han notado pequeñas modificaciones en las propiedades del betún, cuando se hace actuar sobre el mismo, al agente alquilante o acilante, en ausencia de catalizador. Estos pequeños cambios pueden ser una consecuencia de las reacciones de origen térmico que conducirian a isomerizaciones, desalquilaciones y transposiciones aunque de una forma poco acusada, debido a la falta de una actividad catalítica.

4. El proceso de envejecimiento térmico se ha realizado para intentar simular, a nivel de Laboratorio, las transformaciones que tendrá el material cuando se realice su puesta en servicio. Se ha comprobado, que dicho proceso llevó consigo un aumento de la rigidez del material, así como de su viscosidad aparente.

5. El betún de partida era un newton puro desde el punto de vista reológico; los nuevos productos obtenidos son ya de carácter pseudo-plástico (véanse los diagramas de las figuras 1, 2 y 3 ). Dicho alejamiento de la fluencia newtoniana se hace más acusado a medida que disminuye la temperatura.

6. El coeficiente de susceptibilidad térmica obtenido a partir de las pendientes viscosidad-temperatura, en papel dobie logarítmico, disminuye en valor absoluto con relación al betún original. Por tanto la susceptibilidad térmica, como se puede comprobar en la tabla IX ha sido disminuida, de una manera notable, al transformar el betún por reacción de Friedel y Crafts. 


\section{B I B LIOGRAF I A}

(1) ORTEGA, J. J.; BLANCO, M. y CUEVAS, A.: "Modificación de los ligantes bituminosos para su empleo en pavimentos flexibles". Trab. Tec. Inst. Bras. de Petróleo., 696-716. Rio de Janeiro, Dic. 1981.

(2) YEN, T. F.: Amer. Chem. Soc. Div. Pet. Chem. Prepr. 77-4-F102. New York. 1972.

(3) EPINAT, M.: "Preparation de nouveaux liants á base de bitumes en vue d'applications routiéres". Thése doct-ing. Universidad de Burdeos, 1975.

(4) ABINADER, N.: "Modifications chimiques des bitumes pétroliers en vue d'applications routiéres" Thése 3e cycle. Universidad de Burdeos, 1975

(5) EPINAT, M.: Bull. Liaison Labo. P. et Cha., spécial V., 109-118, Diciembre 1977.

(6) BLANCO, M.; ESTEBAN, S.; MARINAS, J. M.; MARTINEZ, M. P. y PEREZ-OSSORIO, R.: Acta Port. Vol. 2. 323. Lisboa, 1979.

(7) ALBEROLA, A.; BLANCO, M.; MARINAS, J. M. y PEREZ-OSSORIO, R.: "Ortofosfatos de aluminio tipo Kearby, como catalizadores de Friedel y Crafts. II Alquilación, isomerización y desalquilación". Comunicación presentada al IV Congreso Iberoamericano de Catálisis. Méjico D.F. 1974.

(8) A.S.T.M. D-5-25; A.S.T.M. D. 36-66T; N.L.T.-182/68 y A.S.T.M. D 113-44.

(9) PINILLA, A.: Alemas, 54, 35-47 (1971).

\section{publicaciones del i.e.t.c.c.}

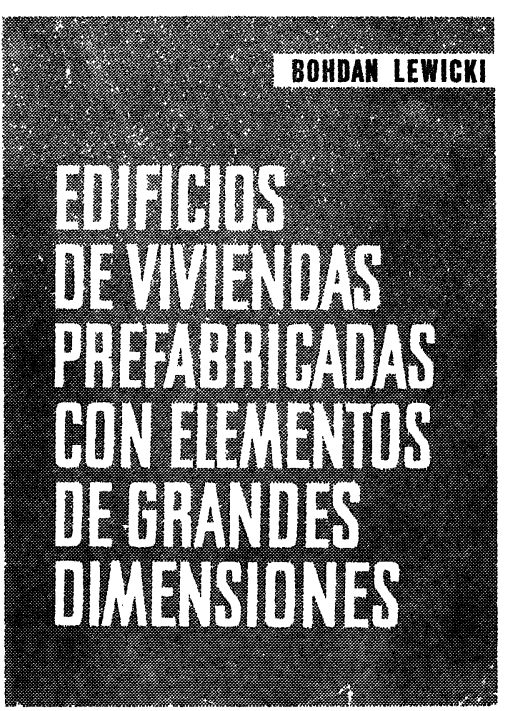

Bohdan Lewicki

Este libro trata de los problemas relativos a la construcción de los edificios de viviendas o publicos realizados con elementos prefa o publicos realizados con elementos prefa-
bricados de grandes dimensiones. Se han estudiado los problemas de arriostramiento, asi como los que plantea la resistencia de los elementos y de la estructura; se han examinado las cuestiones de orden higrotermico, acústico y de resistencia al fuego; también se ha profundizado en el estudio de la estanquidad de los muros exteriores y de las juntas.

La obra incluye numerosas ilustraciones que dan detalles de diversas soluciones, asi como ejemplos de cálculo, tablas de valores numéricos, diagramas y ábacos.

Un volumen encuadernado en tela, de $24 \times 17 \mathrm{~cm}$, compuesto de 616 págs.

Precios: 2.500 ptas.; \$USA 36.00 .
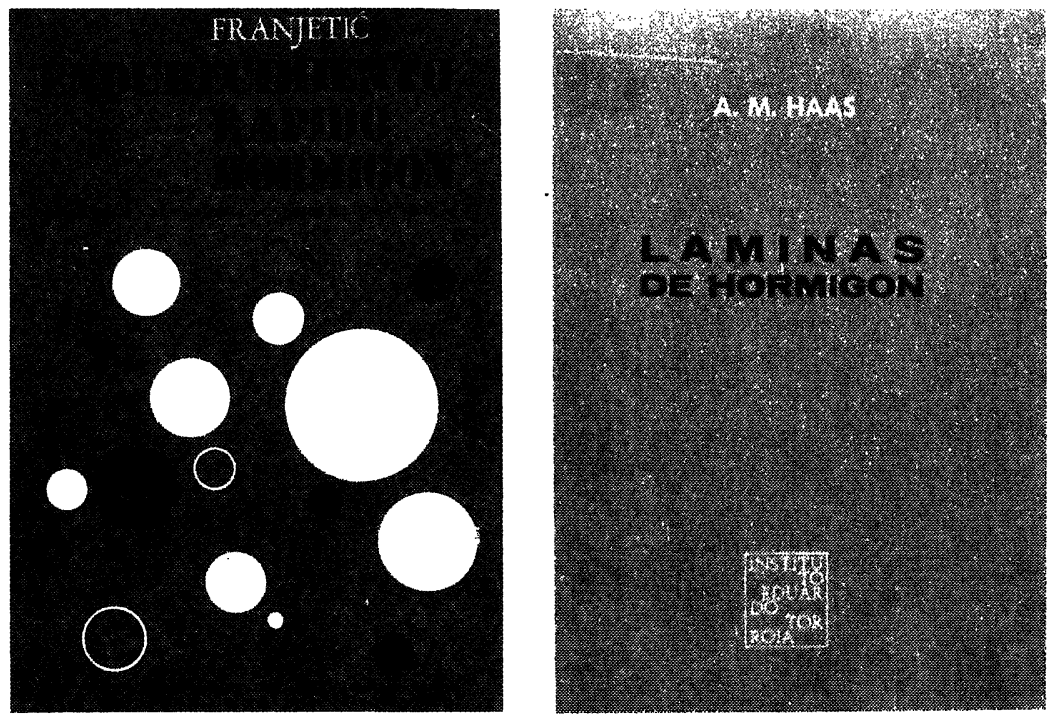

Zorislav Franjetić

En la obra de Franjetić se expone de una forma minuciosa, ordenada y sistemática, todo un cuerpo de doctrina que reúne el cotodo un cuerpo de doctrina que reúne el conocimiento actual sobre el endurecimiento
rápido del hormigón. Parte el autor de los rápido del hormigón. Parte el autor de los
principios básicos y llega a las últimas consecuencias y realidades técnicas y econó micas.

Es una obra de consulta, tanto para el investigador sobre la materia, como para el proyectista y el realizador y montador de plantas e instalaciones y equipos de curado y endurecimiento rápido.

Un volumen encuadernado en cartóné, de $17 \times 24,5 \mathrm{~cm}$, compuesto de 385 págs. 110 figuras y 10 tablas.

Precios: 2.500 ptas.; \$USA 36.00 .

\section{A. M. Haas}

Al escribir este libro el autor intento poner a disposición de los estudiantes y de los ingenieros unos conocimientos prácticos, adecuados para servir de guia en el diseño y construcción de láminas delgadas de hormigón.

El autor está convencido de que el éxito en el diseño de una lámina exige, por parte del proyectista, un examen de las tres fases por proyectista, un examen de las tres fases por
las que pasa la materialización de la lámina: el diseño, el análisis estructural y la construcción de la estructura.

Un volumen encuadernado en tela, de $17 \times 24,5 \mathrm{~cm}$, compuesto de 420 págs., 141 figuras, 22 fotografias y 6 tablas.

Precios: 2.500 ptas.; SUSA 36.00 